\title{
Laboreal
}

Volume $17 \mathrm{~N}^{\circ} 1$ | 2021

Trabalhar hoje: mudanças, permanências, estratégias, reinvenções

\section{8 de abril, Dia Mundial em Memória às Vítimas de Acidentes e Doenças do Trabalho : o que aprender com a COVID-19?}

28 de abril, Día Mundial de la Seguridad y la Salud en el Trabajo : ¿qué aprender

del COVID-19?

28 avril, Journée Mondiale de la Sécurité et de la Santé au Travail : que faut-il apprendre du COVID-19?

April 28, International Workers' Memorial Day : what to learn from COVID-19?

\section{Élida Azevedo Hennington}

\section{(2) OpenEdition}

\section{Journals}

Edição electrónica

URL: https://journals.openedition.org/laboreal/17665

DOI: $10.4000 /$ laboreal. 17665

ISSN: 1646-5237

Editora

Universidade do Porto

Refêrencia eletrónica

Élida Azevedo Hennington, «28 de abril, Dia Mundial em Memória às Vítimas de Acidentes e Doenças do Trabalho : o que aprender com a COVID-19?», Laboreal [Online], Volume 17 № 1 | 2021, posto online no dia 18 junho 2021, consultado o 20 junho 2021. URL: http://journals.openedition.org/laboreal/ 17665 ; DOI: https://doi.org/10.4000/laboreal.17665

Este documento foi criado de forma automática no dia 20 junho 2021.

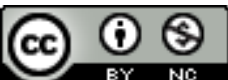

Laboreal está licenciado com uma Licença Creative Commons - Atribuição-NãoComercial 4.0 Internacional. 


\title{
28 de abril, Dia Mundial em
} Memória às Vítimas de Acidentes e Doenças do Trabalho : o que aprender com a COVID-19?

\author{
28 de abril, Día Mundial de la Seguridad y la Salud en el Trabajo : ¿qué aprender \\ del COVID-19? \\ 28 avril, Journée Mondiale de la Sécurité et de la Santé au Travail : que faut-il \\ apprendre du COVID-19? \\ April 28, International Workers' Memorial Day : what to learn from COVID-19?
}

\section{Élida Azevedo Hennington}

1 Em 2003, a Organização Internacional do Trabalho (OIT) reforçou a histórica atuação de trabalhadores e entidades sindicais em defesa do trabalho decente, seguro e saudável, instituindo o dia 28 de abril como o Dia Mundial de Segurança e Saúde no Trabalho, em memória às vítimas de acidentes e doenças ocupacionais (OIT, 2021). Nesse dia, são celebrados eventos no mundo todo para a mobilização de trabalhadores, empregadores e governantes em torno do tema dos riscos de acidentes e doenças relacionados ao trabalho, bem como de suas graves consequências. O Dia Nacional em Memória das Vítimas de Acidentes e Doenças do Trabalho foi instituído no Brasil, há 16 anos, com a Lei $11.121 / 2005$.

2 Em relação à morbimortalidade ocupacional, estimativas da OIT apresentam dados alarmantes, como a ocorrência anual de 2,3 milhões de óbitos no mundo resultantes de acidentes ou doenças ocupacionais, com aproximadamente 6.400 pessoas mortas diariamente, de acordo com essas estatísticas (ILO, 2015).

Durante a conferência internacional da OIT em 2019, reunião anual que define as normas internacionais do trabalho e as políticas gerais desta agência das Nações Unidas, os sindicatos reivindicaram o reconhecimento de que a saúde e a segurança no trabalho devem ser inseridas nos princípios e direitos fundamentais da Organização - 
os direitos decentes, universalmente aceitos e vinculantes, que protegem todos os trabalhadores, em todos os lugares. A Declaração do Centenário da OIT reconheceu que "condições de trabalho seguras e saudáveis são fundamentais para um trabalho decente" (ILO, 2019).

4 Hoje, as doenças representam a principal causa de óbito relacionado ao trabalho, matando quase seis vezes mais trabalhadores do que os acidentes - típicos e de trajeto. Em relação à morbidade, estima-se a ocorrência anual de 160 milhões de casos de doenças ocupacionais no mundo. Segundo a OIT, isso demonstra a necessidade de um novo paradigma de prevenção: aquele que também se concentra nas doenças relacionadas ao trabalho, e não apenas nos acidentes (ILO, 2015).

O alastramento da COVID-19 pelo planeta e suas repercussões no mundo do trabalho, após a identificação e divulgação dos primeiros casos ainda em 2019, parecem confirmar esse novo paradigma.

\section{A pandemia pela COVID-19 e o mundo do trabalho}

6 A pandemia do novo coronavírus resultou numa grave crise humanitária global, que afetou diretamente trabalhadores em todas as partes do mundo. Demonstrou, de forma aguda, a crescente fragilidade tanto dos mecanismos de assistência e promoção da saúde dos trabalhadores, quanto de prevenção de acidentes e adoecimentos relacionados ao trabalho, escancarando o esgarçamento progressivo da rede de proteção social e de direitos. Nesse contexto, houve um forte clamor dos trabalhadores pela classificação da COVID-19 como doença ocupacional durante o Congresso da International Trade Union Confederation (ITUC, 2020).

7 A falta de proteção à saúde dos trabalhadores exposta dramaticamente pela pandemia em várias partes do mundo ditou o tema da campanha de 2021 : "Saúde e segurança são um direito fundamental no trabalho". De acordo com a OIT, são considerados direitos fundamentais e de mais alto grau de comprometimento e responsabilidade por parte dos governos : a liberdade de associação e o direito à negociação coletiva ; a eliminação do trabalho forçado e do trabalho infantil; e o fim da discriminação no emprego/ ocupação. As entidades sindicais reivindicam a inclusão de mais um direito que reforce a necessidade de proteção efetiva nos locais de trabalho e atenção à saúde dos trabalhadores (ITUC, 2021).

8 Parece, então, que a evolução da pandemia no mundo só fez reforçar o motivo pelo qual a saúde e a segurança devem ser um direito de todos os que trabalham. E, de fato, a eclosão de surtos de COVID-19, atingindo diferentes categorias profissionais e em distintos processos e locais de trabalho, tal como o crescente número de adoecimentos e mortes de trabalhadores da linha de frente no atendimento à população, considerados "essenciais", acendeu o sinal de alerta.

9 Na verdade, a demora em prover boas condições de trabalho, equipamentos de proteção adequados e suficientes e promover o registro desses eventos como acidente do trabalho, ou mesmo de reconhecer direitos desses trabalhadores e de seus familiares, só vieram confirmar, de forma grave, abrupta e em grande escala, a paulatina e crescente transformação e crise no mundo do trabalho, agravadas por políticas neoliberais, num processo incessante de precarização e perda de direitos ao longo do tempo. 
10 A evolução da pandemia levou governos, empregadores, trabalhadores e a população em geral a enfrentar desafios sem precedentes. Assim, no que concerne à OIT, decidiuse que o Dia Mundial da Segurança e Saúde no Trabalho focará em estratégias para fortalecer os sistemas nacionais de saúde e segurança, no sentido de construir resiliência às adversidades atuais e futuras, com base nas experiências vividas e lições aprendidas com a COVID-19 (OIT, 2020).

11 Muitas entidades sindicais, pesquisadores e instituições de defesa dos direitos coletivos e individuais na área trabalhista têm indicado a necessidade de resguardar a saúde e garantir direitos aos trabalhadores expostos ao risco de infecção, adoecimento e morte pelo novo coronavírus, bem como assegurar proteção a suas famílias (Alici et al., 2020 ; Carlsten, 2021 ; George \& George, 2020 ; Moen, 2020). A prevenção e a implementação de políticas compensatórias e de proteção à saúde são passos importantes na luta dos trabalhadores por melhores condições de vida e trabalho.

\section{A COVID-19 e o reconhecimento de sua relação com o trabalho}

12 Segundo a ISSA (International Social Security Association) - entidade surgida em 1927 sob os auspícios da OIT e que hoje reúne mais de 300 organizações de seguridade social, em mais de 150 países -, se é verdade que a COVID-19 ainda não consta na lista de doenças profissionais da OIT, como as hepatites B e C e a infecção pelo HIV, pode ser, porém, enquadrada como doença ocupacional causada por agente biológico. Isto, desde que haja uma relação direta estabelecida cientificamente ou determinada por métodos adequados entre a exposição ao agente biológico, a atividade de trabalho e a doença (ISSA, 2020). A evidência científica de que o SARS-Cov-2 causa a COVID-19 já foi estabelecida, mas, em muitos casos, é difícil provar a exposição ocupacional ou que a doença foi contraída em decorrência do trabalho.

13 À medida do avanço da pandemia, países como Noruega, Itália, Alemanha, Canadá, França, EUA e África do Sul reconheceram como ocupacional a doença causada pelo novo coronavírus, com diferenças na caracterização e considerando como categorias afetadas especialmente médicos, enfermeiros e outros profissionais da área da saúde (Chirico \& Magnavita, 2020 ; Godderis et al., 2020 ; ISSA, 2020 ; OSHA, 2020/2021).

14 O médico italiano Antonio Mutti afirmou, inclusive, que a COVID-19 representa uma nova doença ocupacional, a mais mortal dos últimos tempos (Mutti, 2020). Saliente-se que, desde o primeiro surto em Wuhan, a maioria dos casos foi de origem ocupacional, atingindo, em primeiro lugar, pessoas que trabalhavam no mercado atacadista de frutos do mar e outros animais. Posteriormente, profissionais de saúde tornaram-se grupo de alto risco : do total de 138 pacientes tratados em um hospital de Wuhan, 40 pacientes ( $29 \%$ dos casos) eram profissionais de saúde - 31 dos quais (77,5\%) trabalhavam em enfermarias gerais, $7(17,5 \%)$ no pronto-socorro e $2(5 \%)$ na unidade de cuidados intensivos (UTI).

Deve-se destacar que, em tese, os trabalhadores na linha de frente da resposta à pandemia estão claramente mais expostos. E não somente ao perigo de infecção pelo novo coronavírus, à morte, ao adoecimento e às eventuais sequelas, mas também outros prejuízos à saúde, como, por exemplo: fadiga pelas longas horas de trabalho, sofrimento psicológico, síndrome de burnout e diversos problemas de saúde mental, 
devido ao estresse crônico/experiências traumáticas, estigma e violência (Godderis et al., 2020 ; Mutti, 2020 ; WHO, 2020). Como constataram Burdorf et al. (2020), a COVID-19 constitui um enorme desafio, posto que trabalhadores em muitas outras ocupações, além dos profissionais de saúde, enfrentam alto risco de exposição ao vírus. Há, efetivamente, uma longa lista de ocupações que envolvem contato direto com o público e proximidade física com outras pessoas, tais como barbeiros, manicures, trabalhadores do comércio, de lojas, bares, restaurantes e serviços de entrega, etc. Por outro lado, existem atividades em que os trabalhadores, ao contrário dos considerados legalmente "essenciais", têm a liberdade de trabalhar a partir de casa, reduzindo consideravelmente o risco de infecção.

$\mathrm{Na}$ Itália, por conta dos debates sobre essa matéria, hipóteses interessantes têm emergido. $O$ estudo de Marinaccio et al. (2020), ao investigar os pedidos de indenização de trabalhadores naquele país em virtude da infecção pelo novo coronavírus, identificou que a COVID-19 foi adquirida no local de trabalho em uma parte substancial de casos $(19,4 \%)$. Os principais setores econômicos atingidos envolviam atividades relacionadas à saúde humana e ao serviço social, embora reivindicações de compensação ocupacional também tenham incluído outros trabalhadores de setores essenciais, como fábricas de processamento de carnes e aves, setores de armazenamento, balconistas, carteiros, farmacêuticos e trabalhadores de limpeza. 0 estudo concluiu que é necessário construir um sistema de vigilância em saúde do trabalhador para casos de COVID-19, incluindo uma análise individualizada das circunstâncias em que a infecção é adquirida, para a prevenção do risco ocupacional, orientação e apoio ao sistema de seguro e à gestão das políticas de vacinação.

Isso é particularmente relevante para novas doenças ocupacionais emergentes, como a COVID-19, quando os conhecimentos sobre a doença e os fatores de risco associados são limitados, e a implementação de medidas de prevenção é urgente. Van der Molen et al. (2020) ressaltam que, na Holanda, embora os pedidos de indenização possam ser influenciados pela necessidade financeira do trabalhador e o registro de doenças ocupacionais possa ser enviesado pela atuação de médicos do trabalho, a notificação e análise das informações sobre os casos de COVID-19 relacionados ao trabalho são importantes para identificar grupos mais vulneráveis e explorar o impacto dos fatores de risco.

18 Parece evidente que os trabalhadores - em especial, os trabalhadores ditos essenciais são altamente expostos ao SARS-CoV-2, mais do que a população em geral. Uma equipe de investigadores canadenses (Carlsten et al., 2021) destacou alguns pontos relevantes para a proteção dos trabalhadores que atuam no enfrentamento da pandemia : fatores como baixo nível socioeconômico conferem desvantagem e aumentam a suscetibilidade e vulnerabilidade desses trabalhadores, agravando o impacto da COVID-19, já que, neste caso, o contato com o vírus é praticamente inevitável, e a aplicação dos fundamentos de saúde e segurança do trabalho são críticos.

Seria imprescindível prover um ambiente de trabalho seguro e saudável com o máximo cuidado e a adequada proteção aos trabalhadores, garantindo-lhes medidas de higiene e prevenção da contaminação, como a testagem dos mais expostos, vulneráveis e trabalhadores suscetíveis ; a implementação de planos de saúde e segurança para locais e empregos específicos, com disponibilização de equipamentos de proteção individual e coletiva ; a adoção de medidas de identificação e isolamento de casos de infecção ; o desenvolvimento de sistemas de informação e de vigilância em saúde ; e a disseminação 
pública de dados e informações. Os autores finalizam afirmando que, a partir de agora, temos que estar preparados para outros eventos catastróficos como a pandemia de COVID-19 e que riscos de novos desastres globais não devem ser subestimados (Carlsten et al., 2021).

No caso da Itália, o Instituto Nacional de Seguro de Acidentes de Trabalho (INAIL Istituto Nazionale Assicurazione Infortuni sul Lavoro) afirmou ser indispensável reconhecer a COVID-19 como doença relacionada ao trabalho, não apenas no que se refere a médicos e enfermeiros, mas igualmente em outros trabalhadores da linha de frente, como aqueles que atuam em contato direto com o público (por exemplo, assistentes sociais e funcionários de hospitais, caixas de supermercados e demais trabalhadores do comércio, dentre outros). No caso desse grupo, existe, na Itália, uma presunção legal de doença ocupacional, o que pode permitir a concessão imediata de benefícios de seguro. Em outras situações, todavia, a relação de causa e efeito deve ser demonstrada caso a caso pelo trabalhador que pede a indenização. Deve-se destacar, entretanto, que, dada a presença do vírus não só no ambiente de trabalho, mas também fora dele, provar sua origem ocupacional pode ser impossível para o trabalhador (probatio diabolica, em latim), e o enquadramento adequado de COVID-19 como doença relacionada ao trabalho torna-se um verdadeiro obstáculo (Chirico \& Magnavita, 2020).

\section{A COVID-19 e a saúde do trabalhador no Brasil}

21 Moen (2020) procurou sinalizar, num editorial da revista Occupational Medicine, a importância do reconhecimento mundial da doença causada pelo novo coronavírus como ocupacional, de modo a possibilitar compensações, gerar estudos e análises estatísticas e possibilitar o planejamento de medidas preventivas em saúde e segurança.

No Brasil, assim como em outras partes do mundo, há um movimento em defesa do reconhecimento da COVID-19 como doença relacionada ao trabalho, no caso de trabalhadores que foram obrigados a exercer suas atividades de trabalho fora de seus domicílios (Maeno \& Carmo, 2020). Os argumentos remetiam às situações cotidianas de exposição ao vírus e à facilidade de disseminação da infecção, principalmente por pessoas assintomáticas ou oligossintomáticas. A esse quadro, somam-se a falta de condições de trabalho, de estrutura e de políticas sociais e de controle da pandemia no país; a incapacidade de prover medidas de isolamento e distanciamento social; a exposição forçada ao risco nos ambientes de trabalho, no transporte e vias públicas ; a impossibilidade de exercer o direito legal de recusa a trabalho que julgar de risco grave ou iminente ; dentre outras limitações.

Segundo a legislação previdenciária brasileira, a COVID-19 pode ser reconhecida como doença ocupacional quando esta resulta das condições especiais em que o trabalho é executado e com ele se relaciona diretamente. Pode ainda ser considerada acidente de trabalho diante da hipótese em que a doença seja proveniente de contaminação acidental do empregado pelo vírus SARS-CoV-2, no exercício de sua atividade. Nesse caso, a perícia médica federal deverá caracterizar tecnicamente a identificação do nexo entre o trabalho e o agravo (Ministério da Economia, 2020). Na prática, a COVID-19 pode ser reconhecida como doença ocupacional, na modalidade mesopatia, somente se for comprovado que a contaminação está diretamente relacionada ao trabalho, decorrente das condições especiais em que foi realizado. 
Para Maeno e Carmo (2020), no entanto, o reconhecimento da COVID-19 como doença relacionada ao trabalho no Brasil deve-se dar para todos os trabalhadores expostos, independentemente de vínculo empregatício formal. Desta forma, todos os casos de infecção pelo novo coronavírus em pessoas que precisem sair de casa para trabalhar precisam ser notificados ao Sistema Único de Saúde como doença relacionada ao trabalho, e os trabalhadores do mercado formal e contribuintes do Seguro de Acidente do Trabalho (SAT), ligado ao Instituto Nacional do Seguro Social (INSS), também devem registrar o evento por meio da Comunicação de Acidente de Trabalho (CAT). Vale frisar, que, nos casos de reconhecimento da CoVID-19 como acidente de trabalho, os trabalhadores formais têm alguns direitos: um ano de estabilidade no emprego se ocorrer afastamento por mais de 15 dias ; recolhimento do fundo de garantia durante o período de inatividade laboral ; tempo de afastamento/inatividade laboral considerado para fins de aposentadoria; direito de assistência à saúde e reabilitação no caso de sequelas; entre outros benefícios garantidos pela legislação vigente. Ressalte-se ainda que a falta de emissão da CAT por parte das empresas e a subnotificação de acidentes de trabalho, tanto entre trabalhadores do mercado formal quanto do informal, ainda são grandes entraves à implementação da política de saúde do trabalhador e da trabalhadora no Brasil.

Com o avanço da pandemia, observamos diariamente a morte e o adoecimento de milhares de trabalhadores, formais e informais, e o sofrimento de outras tantas pessoas que, há muito tempo, estão à margem do mercado de trabalho, da produção e do consumo, sem direito a qualquer proteção ou benefício. A pandemia, além de revelar uma crise sanitária e humanitária, aprofundou as iniquidades já existentes.

Evidenciou-se que o trabalho é categoria central nas reflexões sobre a pandemia e permanece como determinante fundamental do processo saúde-doença. Mais do que nunca, os trabalhadores que sustentam a economia e o desenvolvimento dos países precisam lutar e estar unidos em defesa da vida, na busca de melhores condições de trabalho, por renda básica e mais empregos, pelo resgate e pela conquista de direitos trabalhistas e sociais, enfim, por uma vida saudável e digna.

\section{BIBLIOGRAFIA}

Alici, N., Beyan, A., \& Simsek, C. (2020). COVID-19 as an occupational disease. Eurasian Journal of Pulmonology, 22(4), 90-100. https://doi.org/10.4103/ejop.ejop_50_20

Burdorf, A., Porru, F., \& Rugulies, R. (2020). The COVID-19 (Coronavirus) pandemic : consequences for occupational health. Scandinavian Journal of Work, Environment \& Health, 46(3), 229-230. https:// doi.org/10.5271/sjweh.3893

Carlsten, C., Gulati, M., Hines, S., Rose, C., Scott, K., Tarlo, S. M., Torén, K., Sood, A., \& de la Hoz, R. (2021). COVID-19 as an occupational disease. American Journal of Industrial Medicine, 64(4), 227-237. https://doi.org/10.1002/ajim.23222 
Chirico, F., \& Magnavita, N. (2020). COVID-19 infection in Italy : an occupational injury. South African Medical Journal, 110(6), 436. https://doi.org/10.7196/SAMJ.2020.v110i6.14855

George, R., \& George, A. (2020). COVID-19 as an occupational disease ? South African Medical Journal, 110(4), 260. https://doi.org/10.7196/SAMJ.2020.v110i4.14712

Godderis, L., Boone, A., \& Bakusic, J. (2020). COVID-19 : a new work-related disease threatening healthcare workers. Occupational Medicine, 70(5), 315-316. https://doi.org/10.1093/occmed/ kqaa056

International Labour Organization - ILO (2015). Global trends on occupational accidents and diseases. Available on https://www.ilo.org/legacy/english/osh/en/story_content/external_files/fs_st_1ILO_5_en.pdf

International Labour Organization - ILO (2019). Centenary declaration for the future of work adopted by the conference at its one hundred and eighth session. Available on https://www.ilo.org/wcmsp5/ groups/public/@ed_norm/@relconf/documents/meetingdocument/wcms_711674.pdf

International Social Security Association - ISSA (2020, April 2). Can COVID-19 be considered an occupational disease ? Available on https://ww1.issa.int/analysis/can-covid-19-be-consideredoccupational-disease

International Trade Union Confederation - ITUC (2020, April 27). COVID-19 should be classified as an occupational disease. Available on https://www.ituc-csi.org/covid-19-occupational-disease?

lang=en

International Trade Union Confederation - ITUC (2021, February 4). Save lives at work :

International Workers' Memorial Day - 28 April. Available on https://www.ituc-csi.org/save-lives-atwork?lang=en

Maeno, M., \& Carmo, J. C. (2020, May 17). A COVID-19 é uma doença relacionada ao trabalho. Observatório da Medicina. Disponível em http://observatoriodamedicina.ensp.fiocruz.br/acovid-19-e-uma-doenca-relacionada-ao-trabalho-por-maria-maeno-e-jose-carlos-do-carmo

Marinaccio, A., Boccuni, F., Rondinone, B., Brusco, A., D’Amario, S., \& Iavicoli, S. (2020). Occupational factors in the COVID-19 pandemic in Italy : compensation claims applications support establishing an occupational surveillance system Occupational and Environmental Medicine, 77(12), 818-821. https://doi.org/10.1136/oemed-2020-106844

Ministério da Economia (2020). Nota Técnica SEI nº 56376/2020/ME. COVID-19. Nexo com o trabalho à luz da legislação Previdenciária. Medida Provisória $n^{\circ}$ 927, de 2020. Secretaria Especial de Previdência e Trabalho, Secretaria de Previdência, Subsecretaria do Regime Geral de Previdência Social, Coordenação-Geral de Benefícios de Risco e Reabilitação Profissional. https://www.gov.br/ economia/pt-br/centrais-de-conteudo/publicacoes/notas-tecnicas/2020/sei_me-12415081-notatecnica-covid-ocupacional.pdf

Moen, B. (2020). COVID-19 should be recognized as an occupational disease worldwide. Occupational Medicine, 70(5), 299. https://doi.org/10.1093/occmed/kqaa086

Mutti, A. (2020). Occupational Medicine in the time of COVID-19. La Medicina del Lavoro, 111(2), 83-86. https://doi.org/10.23749/mdl.v111i2.9546

Occupational Safety and Health Administration - OSHA (2020/2021). COVID-19 Regulations. United States Department of Labor. https://www.osha.gov/coronavirus/standards

Organização Internacional do Trabalho - OIT (2014). Nota Informativa - Dia Mundial de Segurança e Saúde no Trabalho. https://www.ilo.org/legacy/english/protection/safework/worldday/ products04/factsheet04_por.pdf 
Organização Internacional do Trabalho - OIT (2020). Frente a la pandemia : garantizar la seguridad y salud en el trabajo. https://www.ilo.org/wcmsp5/groups/public/---ed_protect/---protrav/--safework/documents/publication/wcms_742732.pdf

Van der Molen, H. F., Kezic, S., Visser, S., de Groene, G., Maas, J., de Wind, A., \& Tamminga, S. (2020). COVID-19 : what can be learned from notifications of occupational diseases ? Occupational and Environmental Medicine, 78(6), 464. https://doi.org/10.1136/oemed-2020-107121

World Health Organization - WHO (2020). Coronavirus disease (COVID-19) outbreak : rights, roles and responsibilities of health workers, including key considerations for occupational safety and health.

Available on https://www.who.int/docs/default-source/coronaviruse/who-rights-roles-responhw-covid-19.pdf ?sfvrsn =bcabd401_0

\section{AUTOR}

\section{ÉLIDA AZEVEDO HENNINGTON}

http://orcid.org/0000-0001-5280-8827

Centro de Estudos da Saúde do Trabalhador e Ecologia Humana, Escola Nacional de Saúde Pública Sergio Arouca, Fundação Oswaldo Cruz, Rio de Janeiro, Brasil, Rua Leopoldo Bulhões, 1480 Manguinhos, Rio de Janeiro. CEP 21040-360

elida.hennington@ensp.fiocruz.br 